\title{
ANÁLISIS FITOQUÍMICO PRELIMINAR DE HOJAS, TALLOS Y SEMILLAS DE CUPATÁ (STRYCHNOS SCHULTESIANA KRUKOFF)
}

\author{
Preliminary phytochemical analysis of Cupatá (Strychnos schultesiana \\ krukoff) stems and seeds
}

Palabras clave: Strychnos, análisis fitoquímico preliminar, alcaloides.

Keywords: Strychnos, preliminary phytochemical study, alkaloids.

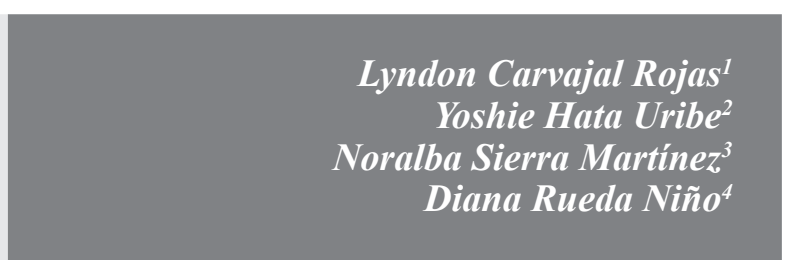

\section{RESUMEN}

Con el fin de ahondar en el conocimiento de la flora colombiana, particularmente aquella presente en la región de los llanos orientales, se eligió la especie Strychnos schultesiana Krukoff para profundizar en su composición química y así establecer una potencial utilidad medicinal o industrial. Para ello se realizó un análisis fitoquímico preliminar de tres de sus órganos -tallos, hojas y semillas-, en el que se evaluó la presencia de los principales grupos de metabolitos secundarios asociados con actividad biológica, a saber: alcaloides, flavonoides, taninos, saponinas, derivados antracénicos, esteroides y/o triterpenoides, cumarinas, glicósidos cardiotónicos y lactonas terpénicas. Se detectó la presencia de alcaloides en gran concentración, lo cual era de esperarse al tratarse de una especie del género Strychnos, ampliamente conocida por su contenido de alcaloides. Es notable también la presencia de flavonoides en los tres órganos. Estos compuestos son ampliamente conocidos por sus propiedades antioxidantes, útiles en la prevención de múltiples enfermedades asociadas a los procesos oxidativos. Los resultados de este trabajo son realmente úti- les como primera aproximación a la ampliación del conocimiento químico y a la posible utilidad de las especies presentes en esta zona de Colombia -con características agronómicas óptimas para el establecimiento de cultivos-, que permitan una utilización racional de los recursos naturales de nuestro país, como fuente de desarrollo regional y nacional.

\begin{abstract}
In order to improve the knowledge of Colombian flora, particularly that belonging to the llanos orientales region, Strychnos schultesiana Krukoff was chosen for a chemical composition in-depth study to establish a potential utility in medicinal or industrial fields. For this analysis, a preliminary phytochemical study of three organs of the plant -steams, seeds and leafs- was made; the purpose was to find the main secondary metabolites associated with biological activities: alkaloids, flavonoids, tannins, saponins, antracenic derivates, steroids, coumarins, cardiotonic glycosides and terpenic lactones. The main compounds were alkaloids. This fact was expected, as this species belongs to
\end{abstract}

1 Ingeniería Forestal, Facultad del Medio Ambiente y Recursos Naturales, Universidad Distrital Francisco José de Caldas. Correspondencia: 1carvajal@udistrital.edu.co.

2 Departamento de Farmacia, Facultad de Ciencias, Universidad Nacional de Colombia, Sede Bogotá. yahatau@unal.edu.co

3 Departamento de Farmacia, Facultad de Ciencias, Universidad Nacional de Colombia, Sede Bogotá. nsierram@unal.edu.co

4 Grupo Búsqueda de Principios Bioactivos, Departamento de Farmacia, Facultad de Ciencias, Universidad Nacional de Co-

lombia, Sede Bogotá. dcruedan@unal.edu.co 
the genus Strhychnos, widely known because of its high alkaloid content. In addition, the existence of flavonoids in the studied organs of the plant is very remarkable. These compounds are well known as scavengers, useful in prevention of diseases related with oxidative processes. Finally, the results of this work are very useful as a first approach to the knowledge and utility of the vegetal species found in this location. Furthermore, this plant has big agronomical advantages related with the culture establishment, which improves the rational use of natural resources in our country, as a means of regional and national development.

\section{INTRODUCCIÓN}

El hombre utiliza las plantas con propósitos medicinales desde tiempos prehistóricos y aún hoy tienen un papel clave en el mantenimiento de la salud de la mayor parte de la población mundial, pese a los avances de la medicina moderna. Esto si se tienen en cuenta las diversas formas en que se utilizan, que van desde la preparación de decocciones e infusiones en zonas rurales y países pobres, pasando por los productos fitoterapéuticos, hasta la obtención de principios activos en países desarrollados para la elaboración de medicamentos. Se estima que en el mundo se utilizan cerca de 10000 especies vegetales con fines medicinales, la mayor parte en sistemas de medicina tradicional (wHo 1993, Calixto 2000, Cañigueral 2002, Newman et al. 2003).

En los países en vías de desarrollo, donde vive el $75 \%$ de la población mundial, se consume menos del $15 \%$ del mercado total de medicamentos. Las plantas medicinales representan, por tanto, el único recurso terapéutico disponible para los sectores más desfavorecidos de esta población. Por lo anterior, las autoridades de salud a nivel mundial han aumentado considerablemente su atención a dichos medicamentos, ya que por un lado constituyen la única medicina disponible en los países en vías de desarrollo y, por otro, se han convertido en una popular alternativa en los países desarrollados (Sharapin 2002).

Para determinar la composición química de las plantas medicinales y conocer sus constituyentes biológicamente activos pueden seguirse metodologías que van desde un análisis fitoquímico prelimi- nar hasta estudios químicos sistemáticos bioguiados. Puesto que este último tipo de estudio requiere una inversión considerable de tiempo y recursos, lo ideal es iniciar con estudios fitoquímicos preliminares que permitan hacer una discriminación de las plantas a estudiar en términos de su composición química, con el fin de seleccionar únicamente aquellas más interesantes para posteriores estudios sistemáticos. El objetivo de un estudio fitoquímico preliminar es determinar la presencia o ausencia de los principales grupos de metabolitos en una especie vegetal, a saber: alcaloides, antraquinonas y naftoquinonas, esteroides y triterpenos, flavonoides, taninos, saponinas, cumarinas, lactonas terpénicas y cardiotónicos. Dado que cada uno de estos grupos de compuestos está relacionado con actividades biológicas específicas, partiendo de los resultados obtenidos en el estudio fitoquímico preliminar es posible orientar investigaciones posteriores para determinar la actividad biológica de las especies en cuestión y los principios activos involucrados.

En el caso del presente análisis fitoquímico preliminar se partió del extracto etanólico de tres órganos -hojas, semillas y tallos- de Strychnos schultesiana (Loganiaceae) (Missouri Botanical Garden 2008), en los cuales se evaluó la presencia de los principales grupos de metabolitos secundarios previamente mencionados, mediante pruebas de tubo y/o cromatografía en capa delgada que se describen de manera detallada en el siguiente apartado.

Las especies del género Strychnos han sido utilizadas tradicionalmente por muchas comunidades indígenas para la preparación de venenos conocidos como "curares". Strychnos schultesiana es una especie trepadora que presenta un comportamiento muy favorable para su manejo y propagación en vivero, de igual manera comienza entre los 3 y 4 años de edad la producción de frutos, muy abundantes en el sitio de recolección y conocidos en la población con los nombres de cupatá, castaña espinosa o solita. Al no conocerse mucho de su composición química, se planteó la necesidad de realización de un análisis fitoquímico preliminar para ahondar en su conocimiento químico.

El cupatá es una planta trepadora de hojas simples, opuestas, sin estipula ni exudado. La corteza exter- 
na es de color café y la viva, amarilla. Las ramas tienen espinas opuestas con las cuales asciende. Flores de color amarillo están dispuestas en cimas axilares. Los frutos son bayas grandes de color amarillo y con numerosas semillas de color curuba que presentan una fragancia muy agradable. En la Figura 1 se pueden observar fotografías de los diferentes órganos de la especie.

La especie se distribuye en Perú, Ecuador y Venezuela. En Colombia la encontramos en el Chocó biogeográfico, Orinoquía, región Andina y piedemonte llanero y es usada por los indígenas de las regiones donde se encuentra para la preparación de curares útiles para capturar las presas fácilmente; para ello untan la punta del dardo o de la flecha con la cual van a herir al animal. Aunque los frutos poseen una pulpa carnosa de un sabor muy agradable, las semillas no se deben consumir ya que contienen estricnina. Con respecto a la propagación, se puede utilizar la semilla de manera exitosa y la planta es de lento crecimiento en sus primeros estados de desarrollo, en los que requiere sombra. Por su carácter ascendente tiende a trepar sobre árboles hasta alcanzar las copas. Se han realizado ensayos de propagación vegetativa que presentan buenos resultados en vivero. La especie presenta rebrotes.
A.

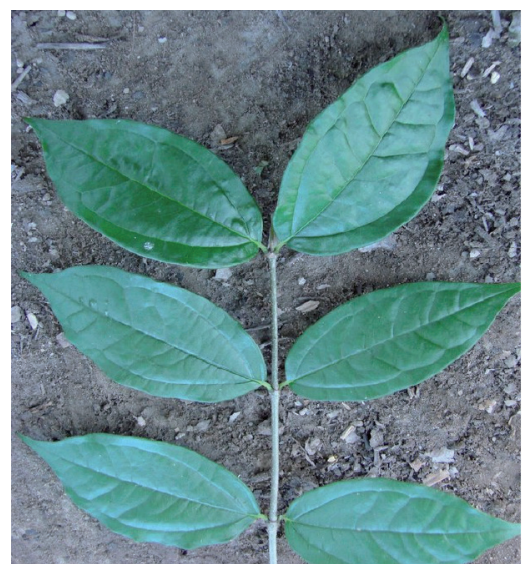



B.
C.

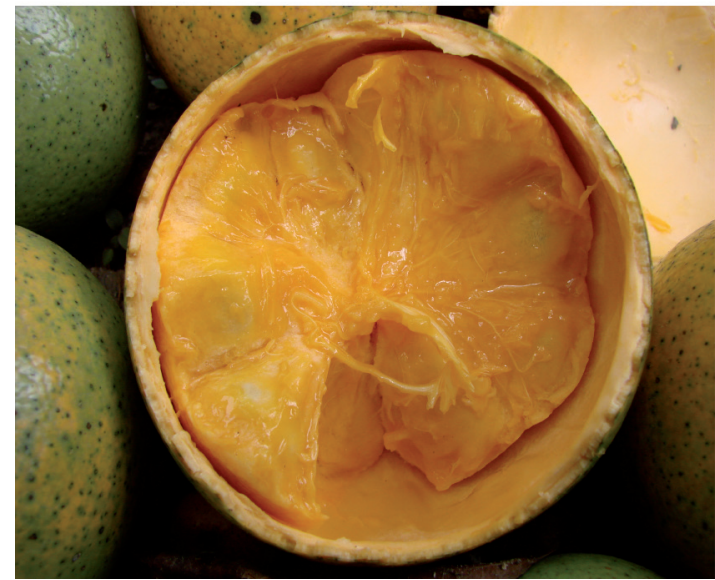

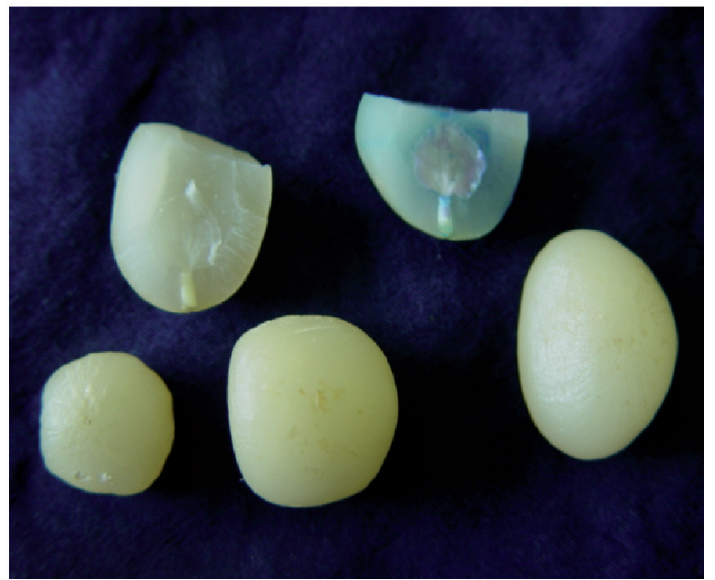

D.

Figura 1. Fotografías de muestras de Strychnos schultesiana A. Hojas, B. Flores, C. Frutos, D. Semillas. 


\section{METODOLOGÍA}

El material vegetal de cupatá fue recolectado en el mes de junio del año 2008, en la finca El Alcaraván, vereda Los Maracos, km 1 vía al Rió Ariari, Granada, Meta. Para realizar el análisis fitoquímico preliminar se utilizó la metodología corrientemente utilizada en el Departamento de Farmacia para tal propósito (Sanabria 1983), la cual se divide en tres etapas: procesamiento del material vegetal, obtención del extracto etanólico y realización de la prueba de identificación de cada uno de los metabolitos.

Procesamiento del material vegetal: Hojas, tallos y semillas de $S$. schultesiana fueron sometidos a un proceso de secado en una estufa de aire circulante a $50^{\circ} \mathrm{C}$ por 48 horas, trabajando cada uno de los órganos de la planta por separado. Posteriormente, el tamaño de partícula se disminuyó pasando el material seco por un molino de discos (hojas y tallos) o un molino de cuchillas (semillas), según el caso.

Obtención del extracto etanólico: 100 g del material vegetal seco y molido de cada uno de lo órganos a estudiar fueron sometidos separadamente a maceración, en un balón de fondo plano por un tiempo de 24 horas, utilizando como solvente de extracción etanol del $96 \%$ en cantidad suficiente para cubrir el material vegetal. Posteriormente se llevó a reflujo por una hora, se dejó enfriar a temperatura ambiente y se filtró y concentró para eliminar el solvente y proceder a realizar las pruebas para cada uno de los metabolitos.

Alcaloides: La mayoría de los alcaloides, con excepción de los alcaloides de amonio cuaternario y $\mathrm{N}$-óxidos de amina, son solubles en solventes orgánicos poco polares, como cloroformo y mezclas de éste, pero pueden formar sales solubles en agua en presencia de ácidos minerales diluidos, como el ácido clorhídrico al 5\% en agua. Esta propiedad ácido-base se aprovechó para su purificación a partir de extractos totales. Luego de este paso inicial se realizaron pruebas de precipitación en medio ácido, utilizando para ello sales de metales pesados como el ioduro de potasio (reactivo de Dragendorff), el ioduro de potasio y mercurio (reactivo de Mayer) y la sal de Reineckato de amonio, entre otros. En la Figura 2 se describe detalladamente el procedimiento de purificación de alcaloides en las muestras evaluadas.

Nafto y antraquinonas: Para la detección de este tipo de metabolitos secundarios se empleó la reacción de Bornträger-Kraus. Para ello, el extracto etanólico seco fue extraído con una solución etanólica en agua (1:7), a la cual se adicionó peróxido de hidrógeno y ácido sulfúrico y se procedió a calentar; bajo estas drásticas condiciones, se hidrolizan los enlaces glicosídicos y se oxidan las antronas y los antranoles hasta antraquinonas, las cuales fueron extraídas con tolueno y agitadas en presencia de una solución de hidróxido de sodio al 5\% que contiene hidróxido de amonio al 2\%. En caso de presencia de nafto o antraquinonas, al dejar separar las fases la capa alcalina (inferior) toma una coloración que va del rosado al rojo intenso, dependiendo de la concentración de estos compuestos en la muestra.

Esteroides y triterpenoides libres: Para su análisis preliminar en plantas la prueba más comúnmente usada es la de Liebermann-Burchard. Para realizar esta prueba, fue necesario hacer un procedimiento de separación previo en el cual se obtuvo un extracto en éter de petróleo a partir del extracto etanólico seco. Este extracto etéreo se extrajo de nuevo con una mezcla de metanol - agua 9:1, se recuperó la capa superior y se sometió a cromatografía en capa delgada, empleando gel de sílice $\mathrm{F}_{254}$ como fase estacionaria y una fase móvil compuesta por una mezcla hexano - acetato de etilo (95:5). La cromatografía desarrollada se dejó secar antes de revelarla, se asperjó con el reactivo de Liebermann-Burchard (solución etanólica de anhídrido acético en presencia de ácido sulfúrico) y se sometió a calentamiento $\left(110^{\circ} \mathrm{C}\right)$ durante 5 a $10 \mathrm{~min}$. Se considera resultado positivo si bajo estas condiciones aparecen manchas en cualquier tonalidad del rojo, azules o verdes.

Flavonoides: Para su detección se emplea principalmente la reacción de la cianidina, conocida también como reacción de Shinoda. Para ello el extracto etanólico seco se extrajo con una solución etanólica en agua (1:7) y se filtró. Este filtrado, denomina- 
do "A", se puso en un tubo de ensayo con $0.5 \mathrm{~g}$ de magnesio en polvo. Seguidamente se adicionó HCL concentrado, gota a gota, hasta el desprendimiento de hidrógeno. Si en estas condiciones se observa la aparición de coloración rojiza, violeta o naranja, se considera positivo para compuestos con el núcleo de la $\gamma$-benzopirona (flavonas, flavonoles, flavanonas, flavanonoles, isoflavonoides y xantonas).

También existe otro tipo de flavonoides denominados leucoantocianidinas, las cuales por cambio en el $\mathrm{pH}$ se tornan de incoloras a intensamente coloreadas de rojo. Para detectar la presencia de este tipo de compuestos en la muestra, el filtrado A fue transferido a un tubo de ensayo, se adicionó ácido clorhídrico concentrado y se sometió a calentamiento en un baño maría hirviendo durante 10-15 min. La aparición de coloración rojiza bajo estas condiciones indica la presencia de leucoantocianidinas.

Taninos: Estos polifenoles tienen la propiedad de unirse a las proteínas y precipitarlas. Por esta razón, la prueba más empleada para la detección de este grupo de metabolitos secundarios emplea el reactivo de gelatina-sal, el cual produce un precipitado blanco en presencia de taninos, luego de haber extraído el extracto etanólico total con una solución acuosa de etanol (7:1). Estos precipitados formados como consecuencia de la presencia de taninos deben ser solubles en urea $10 \mathrm{M}$ y producir coloraciones verdes, azules o negras tras la adición de cloruro férrico al $10 \%$ en agua.

Saponinas: Son glicósidos cuya aglicona consiste en un núcleo esteroidal o triterpénico; esta característica estructural les confiere un carácter anfótero que les permite actuar como tensioactivos. Aprovechando esta propiedad, las dos pruebas más empleadas en la detección de saponinas son la de hemólisis y la de formación de espuma, puesto que al ser tensioactivas las saponinas inestabilizan la membrana celular de los eritrocitos, induciendo su ruptura.

Para realizar la prueba de hemólisis el extracto etanólico fue extraído con la solución acuosa (7:1). En caso de ausencia de taninos, se procedió a realizar las pruebas; de lo contrario, con el fin de eliminarlos, se adicionaron aproximadamente $2 \mathrm{~g}$ de óxido de magnesio, evaporando a sequedad y extrayendo luego con etanol al 96\%. Una vez eliminados los taninos, $1 \mathrm{ml}$ de esta solución se puso en un tubo de ensayo en presencia de $5 \mathrm{ml}$ de suspensión normalizada de glóbulos rojos. Como patrón de hemólisis positiva se emplearon $0.5 \mathrm{ml}$ de una solución de digitonina (10 mg en $100 \mathrm{ml}$ de etanol del 80\%) en presencia de $5 \mathrm{ml}$ de suspensión normalizada de glóbulos rojos y como blanco se emplearon $5 \mathrm{ml}$ de la misma suspensión con $1 \mathrm{ml}$ de agua. La prueba de hemólisis es considerada positiva cuando en 10 min se presenta hemólisis en el patrón y en la muestra, mientras que el blanco permanece sin hemolizar.

La prueba de formación de espuma consiste en agitar vigorosamente la solución acuosa $(7: 1)$, obtenida del extracto etanólico total, en un tubo de ensayo y observar la espuma formada. Ésta debe ser estable por lo menos 30 minutos para poder establecer la presencia de saponinas.

Glicósidos cardiotónicos: Estos compuestos poseen características estructurales similares a las de las saponinas, con una estructura compuesta por un núcleo esteroidal glicosilado (2 desoxiazúcares) y una lactona insaturada de 5 o 6 miembros. Para la detección de éstos pueden emplearse pruebas en tubo de ensayo específicas para cada una de las partes que componen la molécula, o técnicas cromatogáficas. El presente análisis fitoquímico preliminar empleó cromatografía en capa delgada (CCD), sirviéndose de dos placas cromatográficas eluídas en la misma fase móvil (cloroformo: metanol: agua 82:17:1) con el fin de comparar los valores de $\mathrm{Rf}$ de los compuestos revelados. Una de ellas se reveló con el reactivo de vainillina - ácido ortofosfórico (1\% vainillina y $10 \%$ de ácido en etanol), específico para esteroides y triterpenos, con el fin de detectar el núcleo esteroidal de los cardiotónicos. La otra placa se reveló con la reacción de Raymond, la cual utiliza m-dinitrobenceno (2\% en etanol) у маон al $20 \%$; en estas condiciones, las $\gamma$-lactonas $\alpha-\beta$ insaturadas producen coloraciones violeta que desaparecen rápidamente. De esta manera, sólo aquellas manchas que revelan en las dos placas pueden considerarse cardiotónicos pues poseen el núcleo esteroidal además de la lactona insaturada. Esto se evidencia claramente con un patrón de digitoxina. 


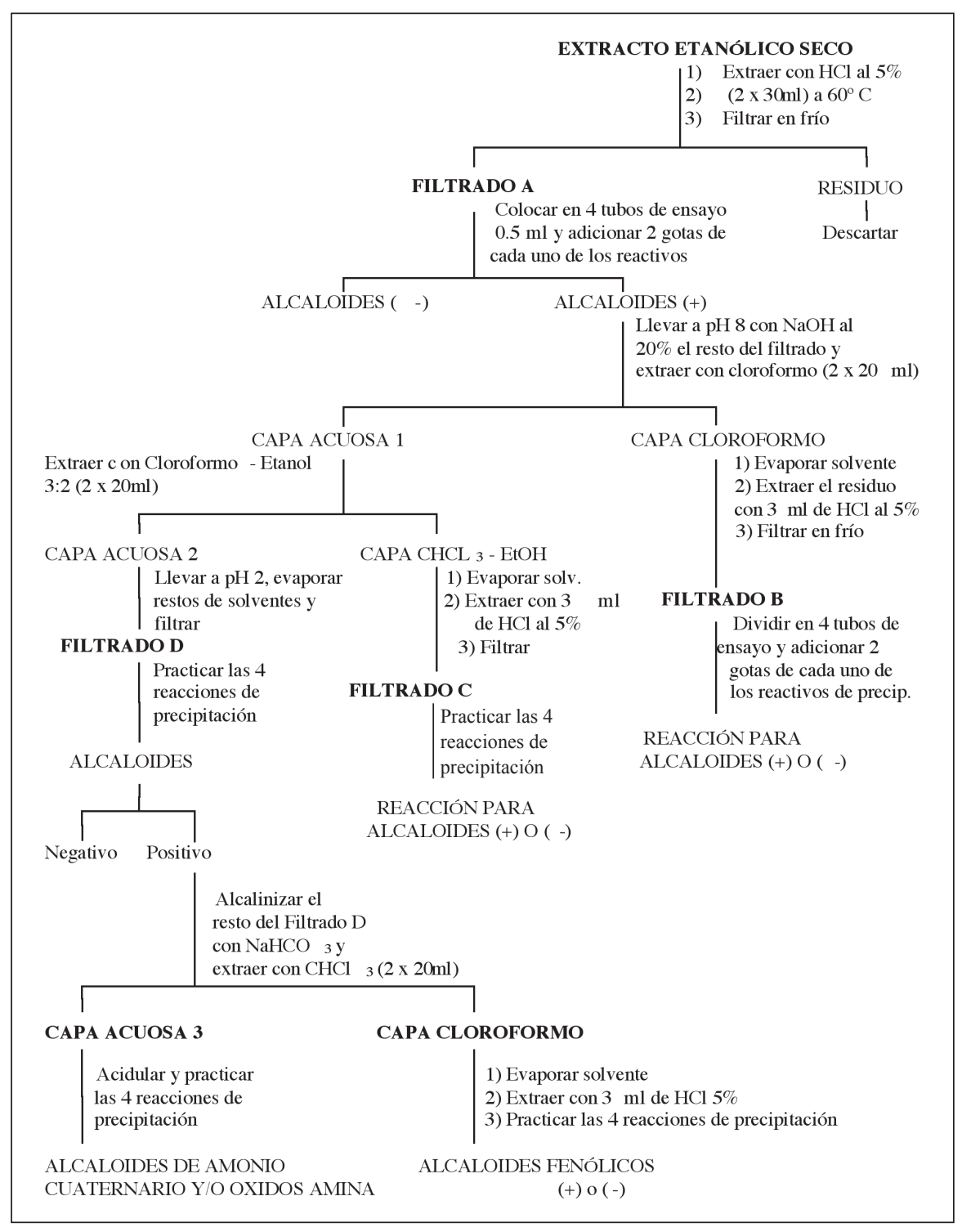

Figura 2. Análisis preliminar de alcaloides.

Previo a la realización de la cromatografía es necesario eliminar las clorofilas del extracto, ya que intervienen con el ensayo. Es necesario hacer lo mismo en el caso del análisis de cumarinas y lactonas sesquiterpénicas. Para ello el extracto etanólico seco fue tratado con un volumen aproximadamente igual de una solución de acetato de plomo al 4\% que contenía $0.5 \%$ de ácido acético, con el fin de precipitar las clorofilas presentes. Esta mezcla se dejó en reposo durante aproximadamente $15 \mathrm{~min}$ y se filtró a presión atmosférica. El filtrado así obtenido fue concentrado a $3 / 4$ de su volumen en eva- porador rotatorio, se dejó enfriar y se extrajo en un embudo de decantación con 2 volúmenes de 30 $\mathrm{ml}$ de cloroformo. Se combinaron las capas clorofórmicas, se adicionó sulfato de sodio anhidro, se filtró y se concentró a $3 \mathrm{ml}$ aproximadamente, obteniendo así la solución a ser utilizada en las pruebas cromatográficas.

Cumarinas: Son compuestos derivados de la $\alpha$ benzopirona. Dado que en su estructura presentan un gran número de instauraciones, estos compuestos exhiben una fuerte fluorescencia azul o verde al ser irradiados con luz ultravioleta, propiedad 
que se aprovecha para su detección. Adicionalmente, puesto que todas las cumarinas poseen en su estructura una $\gamma$-lactona, pueden identificarse mediante las reacciones propias para lactonas. Por este motivo, con el fin de determinar la presencia de cumarinas en la muestra, se desarrolló una cromatografía en capa delgada empleando gel de sílice $\mathrm{F}_{254}$ como fase estacionaria y una mezcla cloroformo-cetona 9:1 como fase móvil. Una vez eluída la placa, se observó a la luz Uv (365 y $254 \mathrm{~nm}$ ) antes de revelarse con la reacción del hidroxamato férrico, específica para lactonas. Esta reacción consiste en asperjar la placa con una mezcla de volúmenes iguales de clorhidrato de hidroxilamina al $2 \%$ en etanol y $\mathrm{NaOH} 2 \mathrm{~N}$, calentar por $5-10 \mathrm{~min}$ a $100^{\circ} \mathrm{C}$ $\mathrm{y}$, al cabo de este tiempo, dejar enfriar y asperjar con volúmenes iguales de HCL $2 \mathrm{~N} \mathrm{y} \mathrm{FeCl}_{3}$ al $1 \%$ en etanol. Aquellos compuestos con fluorescencia al ultravioleta que luego de la aplicación del reactivo revelador exhiben coloración anaranjada al visible son considerados cumarinas. Una placa cromatográfica adicional puede asperjarse con кон al 5\%, el cual acentúa fuertemente la fluorescencia de estos compuestos al ser expuestos a la luz UV.

Lactonas sesquiterpénicas: Estos compuestos, como su nombre lo indica, son terpenos con un esqueleto de 15 átomos de carbono, que tienen además en su estructura una lactona. Para identificar estos compuestos se hicieron dos cromatografías en capa delgada (fase estacionaria: gel de sílice $\mathrm{F}_{254}$ ) eluídas con una fase móvil compuesta por cloroformo y acetona (9:1). Una de las cromatografías fue revelada para terpenos, como se indicó en el caso de los glicósidos cardiotónicos, y la otra se derivatizó para lactonas con el reactivo de hidroxamato férrico, como se indicó en el caso de las cumarinas. Ambas pruebas deben ser positivas para poder concluir que la muestra presenta este tipo de metabolitos secundarios.

\section{RESULTADOS}

En la Tabla 1 se presentan los resultados obtenidos luego de hacer el análisis fitoquímico preliminar de los tres órganos seleccionados de S. schultesiana.

\section{DISCUSIÓN}

En el presente análisis fitoquímico preliminar de hojas, tallos y semillas de la especie $S$. schultesiana se partió de extractos etanólicos puesto que este solvente tiene la capacidad de extraer compuestos de una amplia gama de polaridades, además de ser menos costoso y tóxico que otros solventes orgánicos. La metodología seguida para este análisis fue la previamente estandarizada por Sanabria (1983), y contempla la detección de los metabolitos secundarios generalmente relacionados con actividades biológicas.

A continuación se presenta la discusión de los resultados consignados en la Tabla 1, en relación a los órganos de la especie $S$. schultesiana evaluados.

Con respecto a los alcaloides, fue el grupo de metabolitos secundarios más abundante encontrado en los tres órganos. Sin embargo, dado que la cantidad de precipitado obtenido es proporcional a la concentración de alcaloides en la muestra, puede inferirse que las hojas son el órgano de la planta donde tiene lugar la mayor acumulación de alcaloides, aunque cabe aclarar que en tallos y semillas también se encuentran en concentración apreciable.

Es conocido que las especies del género Strychnos presentan una alta concentración de alcaloides de tipo indólico (estricnina, Yang \& Yang 1993, Santos et al. 2006), los cuales son solubles principalmente en solventes de baja polaridad. Por este motivo, los extractos obtenidos con este tipo de solventes durante el proceso de purificación (filtrados A y B) presentaron una mayor concentración de alcaloides, tal como se muestra en la Tabla 1. Además de alcaloides de tipo indólico, puede inferirse la presencia de alcaloides de amonio cuaternario o $\mathrm{N}$-óxidos de amina en las semillas y las hojas de esta planta, puesto que se obtuvo resultado positivo con el Reineckato de amonio, tanto en el extracto inicial como en la fase más polar del proceso de purificación, donde se encuentra concentrado este tipo de alcaloides. Sin embargo, se deduce que la concentración de éstos es muy baja, lo que se evidencia en la reducida cantidad de precipitado obtenido. 
Tabla 1. Resultados de los análisis fotoquímicos preliminares de hojas, tallos y semillas de Strychnos schultesiana.

\begin{tabular}{|c|c|c|c|c|}
\hline \multirow{2}{*}{\multicolumn{2}{|c|}{ Metabolito }} & \multicolumn{3}{|c|}{ Órgano } \\
\hline & & Hojas & Tallos & Semillas \\
\hline \multirow{6}{*}{ Alcaloides } & Filtrado A & +++ & + & ++ \\
\hline & Filtrado B & +++ & + & ++ \\
\hline & Filtrado C & ++ & + & + \\
\hline & Filtrado D & ++ & - & + \\
\hline & Capa acuosa 3 & + & - & + \\
\hline & Capa Cloroformo & - & - & - \\
\hline \multicolumn{2}{|c|}{ Nafto/Antraquinonas } & - & - & - \\
\hline \multicolumn{2}{|c|}{ Esteroides y/o triterpenoides libres } & ++ & ++ & ++ \\
\hline \multicolumn{2}{|l|}{ Flavonoides } & ++ & ++ & ++ \\
\hline \multicolumn{2}{|l|}{ Taninos } & + & - & - \\
\hline \multicolumn{2}{|l|}{ Saponinas } & + & - & - \\
\hline \multicolumn{2}{|c|}{ Glicósidos cardiotónicos } & - & - & - \\
\hline \multicolumn{2}{|l|}{ Cumarinas } & - & - & - \\
\hline \multicolumn{2}{|c|}{ Lactonas sesquiterpénicas } & - & - & + \\
\hline
\end{tabular}

+ Presencia escaza, ++ Presencia relativamente abundante, +++ Presencia abundante, - No detectado.

La cromatografía realizada para la detección de esteroides $\mathrm{y} / \mathrm{o}$ triterpenos permite determinar la presencia de estos compuestos en las tres muestras evaluadas, lo que se evidencia en la aparición de diversas manchas de tonalidades azulosas y rojizas. Las hojas presentan un mayor número de estos compuestos en comparación con semillas y tallos. Según lo observado, el lupeol no se encuentra presente en esta planta puesto que en ninguno de los órganos evaluados se observan manchas con un valor de Rf similar al obtenido para este patrón.

El análisis fitoquímico preliminar de hojas, tallos y semillas de $S$. schultesiana permitió comprobar también la presencia de flavonoides en la especie, ya que en los tres órganos evaluados se obtuvieron resultados positivos con la reacción de Shinoda y con la del ácido clorhídrico. Lo anterior indica la presencia de flavonoides tipo flavona, flavonol, flavanona, flavanonol, isoflavonoide y leucoantocianidinas.

Por otra parte, puesto que sólo las hojas de $S$. schultesiana ocasionaron precipitación del reactivo gelatina - sal, puede concluirse que en este órgano se concentran los taninos producidos por la planta, y dado que el precipitado obtenido es soluble en urea y produce coloración verde tras la adición de cloruro férrico, se infiere que estos taninos son del tipo catéquico o condensados. Sin embargo, la reducida cantidad de precipitado obtenido indica una baja concentración de taninos en la muestra.

Respecto al análisis de saponinas, sólo el extracto etanólico de las hojas de la especie evaluada produjo hemólisis comparable a la observada con el patrón, mientras que con los extractos de tallos y semillas los eritrocitos permanecieron intactos, lo que se evidenció por la turbidez de la suspensión de glóbulos rojos. De lo anterior se infiere que de los tres órganos analizados, sólo las hojas contienen saponinas. Por otro lado, los resultados de la prueba de espuma para semillas y tallos concuerdan con esta conclusión, puesto que al agitar la muestra no se observó la formación de espuma abundante ni estable; sin embargo, a pesar de los resultados obtenidos en la prueba de hemólisis para el extracto de hojas, el ensayo de espuma arrojó resultados negativos, lo cual puede deberse a que, como se expuso anteriormente, no todas las saponinas tienen la capacidad de producir espuma. Aun con estos resultados a simple vista contradictorios 
para el extracto de hojas, puede inferirse la presencia de saponinas en él, pues la prueba más concluyente para la determinación de saponinas es la de hemólisis.

En cuanto a la detección de glicósidos cardiotónicos, ninguno de los esteroides observados tras revelar una de las placas con vainillina presentó coloración violeta similar a la del patrón en la placa revelada con la reacción de Raymond, por lo cual puede deducirse que la especie $S$. schultesiana no contiene glicósidos cardiotónicos en hojas, tallos ni semillas. Por su parte, el patrón de digitoxina produjo coloraciones violetas, al ser revelado tanto con el reactivo de vainillina en ácido o-fosfórico como con el de Raymond, tal como se esperaba.

Para la detección de cumarinas se emplearon tres fases móviles, de las cuales aquella constituida por tolueno-éter etílico (1:1), saturada con ácido acético al $10 \%$, permitió una mejor separación de los componentes de cada extracto, encontrándose así una mancha fluorescente bien definida en el extracto de tallos, otra en el extracto de semillas y cuatro en el de hojas; sin embargo, al revelar con la reacción del hidroxamato férrico, ninguna de ellas presentó coloración café similar a la del patrón, descartando así la presencia de cumarinas en los extractos.

Para confirmar lo anterior, se asperjó una de las placas desarrolladas en acetato de etilo, con кон al $5 \%$, con lo cual no se intensificó la fluorescencia de las manchas observadas, que incluso desapareció, confirmando que no se trata de cumarinas sino de otros compuestos poliinsaturados que pueden fluorescer también al UV.

Por otro lado, si bien los compuestos fluorescentes presentes en semillas y hojas de $S$. schultesiana no se colorearon tras revelar la placa con la reacción del hidroxamato férrico, los extractos de tallos y semillas presentaron manchas de color café que no fluorescían al uv, lo que indica la presencia de lactonas. Sin embargo, al revelar con el reactivo de vainillina, sólo las lactonas presentes en el extracto de semillas poseen una parte terpénica que se observa de color violeta, mientras que la mancha observada en el extracto de tallos no reveló, por lo que puede concluirse que aunque los tallos poseen lactonas, no son de tipo terpénico.

Para esta especie no se encuentran reportes sobre composición química. Sin embargo, los resultados obtenidos del presente análisis fitoquímico preliminar de hojas, tallos y semillas de esta planta coinciden con los reportados para otras especies del género Strychnos. Ejemplo de lo anterior son los reportes de la presencia de alcaloides en las hojas de $S$. pseudoquina y en semillas de S. nux-vomica (Nicoletti \& Colus 1984, Yang \& Yang 1993, Santos et al. 2006), flavonoides en hojas de $S$. pseudoquina y S. spinosa (Nicoletti et al. 1984, Hoet et al. 2004, Santos et al. 2006) y esteroides y ácidos grasos en raíces y tallos de $S$. nitida (Gu et al. 1997). Adicionalmente, se reporta la presencia de otros grupos de metabolitos no analizados en esta oportunidad, como lignanos en tallos de $S$. vanprukii (Thongphasuk et al. 2004), glicósidos fenólicos, iridoides en madera y corteza de $S$. axillaris (Itoh y Colus 2008) y ácidos orgánicos como el quínico y el logánico y ésteres de los mismos en corteza y madera de S. lucida (Itoh et al. 2008).

A pesar de no haber detectado cumarinas en el presente análisis fitoquímico preliminar de $S$. schultesiana, se ha reportado la presencia de este tipo de metabolitos secundarios en las raíces de $S$. cathayensis (Luo 2006). Sin embargo, este es un grupo de compuestos cuya presencia no se haya ampliamente descrita en la literatura de otras plantas del género Strychnos, por lo que puede tratarse de un grupo poco difundido en estas especies y presente en estos órganos (raíces). Además, cabe aclarar que la presencia de un tipo de metabolito en una planta no condiciona su presencia en otras especies del mismo género.

Grupos de metabolitos secundarios como los glicósidos antracénicos, los cardiotónicos, saponinas y taninos, los cuales no fueron detectados en el presente estudio o sólo fueron detectados en uno de los órganos evaluados, no se encuentran reportados en la literatura consultada para otras especies del género Strychnos. 


\section{CONCLUSIONES}

Los resultados del análisis fitoquímico preliminar efectuado sobre la muestra de hojas de $S$. schultesiana Krukoff evidencian la presencia de alcaloides, taninos, flavonoides, saponinas, esteroides y/o triterpenoides. Los tallos y las semillas de la misma especie presentan alcaloides, flavonoides, lactonas y esteroides. En estos órganos no se detectó la presencia de saponinas ni taninos. Las semillas contienen lactonas terpénicas, mientras que en los tallos se encuentran lactonas pero no de tipo esteroidal. En ninguno de los órganos evaluados se encontró la presencia de cumarinas ni glicósidos cardiotónicos o antraquinónicos.

\section{AGRADECIMIENTOS}

A la Corporación para el Desarrollo del Área del Manejo Especializado la Macarena "CORMACARENA", por la financiación de este proyecto el cual se desarrolló en el marco del convenio 05 de 2001 suscrito con la Universidad Distrital Francisco José de Caldas.

\section{REFERENCIAS BIBLIOGRÁFICAS}

Calixto, J. B. 2000. Efficacy, safety, quality control, marketing and regulatory guidelines for herbal medicines (phytotherapeutic agents). Brazilian Journal of Medical and Biological Research 33: 179-189.

Cañigueral, S. 2002. La fitoterapia: ¿una terapéutica para el tercer milenio? Revista de Fitoterapia (2): 101-121.

Gu, Z., T. Li, P. Xiao, J. Chen \& W. Lian. 1997. Chemical constituents of Strychnos nitida G. Don. Zhongguo Zhong Yao Za Zhi 22 (1): 4041.

Hoet, S., F. Opperdoes, R. Brun, V. Adjakidjé \& J. Quetin-Leclercq. 2004. In vitro antitrypanosomal activity of ethnopharmacologically selected Beninese plants. Journal of Ethnopharmacology 91 (1): 37-42.

Itoh, A., Y. Tanaka, N. Nagakura, T. Nishi \& T. Tanahashi. 2006. A quinic acid ester from Strychnos lucida. J. Nat. Med 60: 146-148.
Luo, T. 2006. Studies on the chemical constituents and biological activities from the stem of Pourthiaea lucida and the root of Strychnos cathayensis. Master's Thesis. Graduate Institute of Pharmaceutical Technology, Tajen University. Taiwan. Disponible en: http://192.192.215.232/ETD-db/ETD-search/ view_etd?URN=etd-0717107-162042

Missouri Botanical Garden. 2008. Disponible en: http://www.tropicos.org/

Nicoletti, M., M. Goulart., R. de Lima, A. Goulart, F. Delle Monache \& G. Marini Bettolo. 1984. Flavonoids and alkaloids from Strychnos pseudoquina. J. Nat. Prod. 47 (6): 953-957.

Newman, D. J., G. M. Cragg \& K. M. Snader. 2003. Natural products as sources of new drugs over the period 1981 - 2002. Journal of Natural Products 66: 1022-1037.

Sanabria, A. 1983. Análisis fitoquímico preliminar. Metodología y su aplicación en la evaluación de 40 plantas de la familia Compositeae. Facultad de Ciencias, Departamento de Farmacia, Universidad Nacional de Colombia. Bogotá.

Santos, F., I. Colus, M. Silva, W. Villegas \& E. Varanda. 2006. Assessment of DNA damage by extracts and fractions of Strychnos pseudoquina, a Brazilian medicinal plant with antiulcerogenic activity. Food and Chemical Toxicology 4 (9): 1585-1589.

Sharapin, N. 2002. Materias primas vegetales para la industria de productos fitofarmacéuticos. Revista de Fitoterapia 1 (3): 23-28.

Thongphasuk, P., R. Suttisri, R. Bavovada \& R. Verpoorte. 2004. Antioxidant lignan glucosides from Strychnos vanprukii. Fitoterapia 75 (7-8): 623-628.

World Health Organization (WHO). 1993. Research guidelines for evaluating the safety and efficacy of herbal medicines. Geneva. Pg. 57.

Yang, X. \& Z. Yan. 1993. Studies on the chemical constituents of alkaloids in seeds of Strychnos nux-vomica L. Zhongguo Zhong Yao Za Zhi 18 (12): 739-40, 763-764. 